\title{
Tactical Decisions for Changeable Cuttlefish Camouflage: Visual Cues for Choosing Masquerade Are Relevant from a Greater Distance than Visual Cues Used for Background Matching
}

\author{
KENDRA C. BURESCH ${ }^{1, *}$, KIMBERLY M. ULMER ${ }^{1}$, CORINNE CRAMER $^{1}$, \\ SARAH MCANULTY ${ }^{1}$, WILLIAM DAVISON ${ }^{1}$, LYDIA M. MÄTHGER ${ }^{1}$, AND \\ ROGER T. HANLON ${ }^{1,2}$ \\ ${ }^{1}$ Program in Sensory Physiology and Behavior, Marine Biological Laboratory, 7 MBL Street, \\ Woods Hole, Massachusetts 02543; ${ }^{2}$ Brown University, Department of Ecology and Evolution, \\ 45 Prospect Street, Providence, Rhode Island 02912
}

\begin{abstract}
Cuttlefish use multiple camouflage tactics to evade their predators. Two common tactics are background matching (resembling the background to hinder detection) and masquerade (resembling an uninteresting or inanimate object to impede detection or recognition). We investigated how the distance and orientation of visual stimuli affected the choice of these two camouflage tactics. In the current experiments, cuttlefish were presented with three visual cues: 2D horizontal floor, 2D vertical wall, and 3D object. Each was placed at several distances: directly beneath (in a circle whose diameter was one body length (BL); at zero BL [(0BL); i.e., directly beside, but not beneath the cuttlefish]; at 1BL; and at 2BL. Cuttlefish continued to respond to 3D visual cues from a greater distance than to a horizontal or vertical stimulus. It appears that background matching is chosen when visual cues are relevant only in the immediate benthic surroundings. However, for masquerade, objects located multiple body lengths away remained relevant for choice of camouflage.
\end{abstract}

\section{Introduction}

Cuttlefish are adept at rapidly altering their body patterning and skin texture for camouflage (Hanlon and Messenger, 1988, 1996; Shohet et al., 2006; Kelman et al., 2007; Mäthger et al., 2007; Allen et al., 2009; Zylinski et al., 2009a, b, c; Barbosa et al., 2012). This expeditious expres-

Received for publication 4 March 2015; accepted 7 May 2015.

* To whom correspondence should be addressed. E-mail: kendraburesch@ gmail.com sion of camouflage body patterns is a visually driven behavior (Holmes, 1940; Hanlon and Messenger, 1988; Marshall and Messenger, 1996) that enables cuttlefish to adjust to homogeneous surroundings (e.g., sand plains) and also complex habitats, such as coral reefs, kelp forests, and temperate rock reefs, with relative freedom from detection or recognition by their many visual predators (Boletzky, 1983; Hanlon, 2007; Hanlon et al., 2011).

Cuttlefish have several camouflage tactics to choose from when trying to avoid visual predation (Hanlon et al., 2009). They primarily deceive predators through background matching, i.e., resembling the background to hinder detection; disruptive patterning, i.e., obscuring edges, shape, and body outline to impede detection or possibly recognition; or masquerade, which is resembling an inanimate or uninteresting object to avoid recognition or detection (e.g., Cott, 1940; Hanlon and Messenger, 1988; Hanlon et al., 2009; Stevens and Merilaita, 2009). The basic camouflage body patterns used by cuttlefish can be grouped into three categories: Uniform, Mottle, and Disruptive (Hanlon, 2007). Each body pattern is made up of light and dark splotches that range along a continuum (Hanlon et al., 2009). The Uniform pattern is composed of small splotches, the Mottle body pattern features medium-sized splotches, and the Disruptive body pattern consists of large splotches along with bars and stripes (Hanlon and Messenger, 1988).

Cuttlefish are benthic animals that dwell primarily on the seafloor, where they use visual information from the surrounding substrate and objects in their immediate vicinity to 
adapt their appearance for camouflage. This information includes horizontal cues, such as sand and gravel, vertical facades (e.g., wall, rock face), as well as discrete, 3D objects (e.g., rocks, algae clumps) (Hanlon and Messenger, 1996; Barbosa et al., 2008a; Buresch et al., 2011). Recent studies have highlighted the importance of vertical features, such as 3D objects and 2D representations of 3D objects, in eliciting the expression of cuttlefish camouflage body patterning (Barbosa et al., 2008a; Buresch et al., 2011; Ulmer et al., 2013). In one set of experiments, cuttlefish preferentially masqueraded as high-contrast, 3D objects that occupied only a small percentage of the visual field in their environment (Buresch et al., 2011). In another experiment, vertical features alone had a stronger influence on body patterning than horizontal, benthic cues (Ulmer et al., 2013).

Although a growing body of literature is beginning to unravel those visual cues that elicit different body patterns for camouflage (e.g., contrast, aspect ratio, shape, substrate intensity, spatial phase, edges, and pattern size; Hanlon and Messenger, 1996; Chiao and Hanlon, 2001a, b; Chiao et al., 2005, 2009, 2010, 2013; Barbosa et al., 2007, 2008a, b; Shohet et al., 2007; Kelman et al., 2008; Hanlon et al., 2009; Zylinski et al., 2009a), the distance at which visual cues are relevant for camouflage has not been studied empirically. This series of experiments addressed two specific questions: (1) How far away from the animal are visual cues relevant for camouflage? and (2) How do 2D and 3D visual stimuli-and their orientation and distance-influence body pattern choice for background matching or masquerade?

\section{Materials and Methods}

\section{Animals}

European cuttlefish (Sepia officinalis Linnaeus, 1758) were hatched, reared, and maintained in the Marine Resources Center facility of the Marine Biological Laboratory in Woods Hole, Massachusetts. Twenty animals (average mantle length $(\mathrm{ML})=5.8 \mathrm{~cm}, \mathrm{SD}=0.5 \mathrm{~cm}$; average White Square area $=2.25 \mathrm{~cm}^{2}, \mathrm{SD}=0.4 \mathrm{~cm}^{2}$ ) were used for these experiments. (For a full description of the White Square, see Chiao and Hanlon, 2001a.) We also measured each animal's body length ( $\mathrm{BL}$; average $\mathrm{BL}=8.2 \mathrm{~cm}, \mathrm{SD}=0.7 \mathrm{~cm}$ ), and used these values as a distance measure for the experimental substrates and objects.

\section{Experimental setup}

Experiments were conducted in a circular, 42-cm diameter tank with flow-through seawater with a depth of $10 \mathrm{~cm}$. The tank was located inside a black tent to prevent disturbances during the experimental trials. A circular, $37 \mathrm{~cm}$ diameter, LED white light source (High Brightness LED strip lights; Environmental Lights, San Diego, CA) was placed directly above the arena to reduce the effect of shadows. To maintain a consistent distance of presented stimuli, cuttlefish were placed in a clear, plexiglass cylinder (14.5 cm diameter, $14.5 \mathrm{~cm}$ height) located in the center of the experimental tank. Animals could rotate within this cylinder, but could not swim a significant distance. Cuttlefish usually settled within this cylinder and showed a stable body pattern within several minutes.

Animals were tested individually, and both animal and stimulus orders were randomized. Animals were given 15-60 min to acclimate to the tank; an animal was considered acclimated when it showed a stable body pattern with little or no motion. Cuttlefish were observed on a TV monitor outside the tent, and images were taken remotely (Canon Rebel XS, Canon U.S.A., Inc., Melville, NY) after the animal had settled. One image was taken for each cuttlefish on each substrate per 3D object combination.

As was shown earlier, cuttlefish use three basic body pattern types for camouflage: Uniform, Mottle, and Disruptive, with variations on each pattern type (Hanlon et al., 2009; for a description of each body pattern type, see Hanlon and Messenger, 1988). In the current experiments, we used large-scale, high-contrast checkerboards, which are known to elicit the Disruptive body pattern, to investigate the body pattern response of $S$. officinalis, because the visual cues used to evoke this pattern have been studied extensively (Chiao and Hanlon, 2001a, b; Mäthger et al., 2006, 2007; Barbosa et al., 2007, 2008a; Chiao et al., 2007, 2009; Kelman et al., 2007; Zylinski et al., 2009a, b). In these experiments, cuttlefish may use Disruptive body patterning to background match the black and white checkerboard on the substrate and/or the wall, or to masquerade as a 3D object.

All stimuli were positioned in the same place for each animal. Artificial substrates and objects were made using uniform gray computer printouts ( $\mathrm{RGB}=142 ; 50 \%$ gray) designed to elicit a Uniform body pattern. Large black $(\mathrm{RGB}=255)$ and white $(\mathrm{RGB}=0)$, high-contrast checkerboard squares were fashioned to elicit a Disruptive body pattern (checkerboard square size $=2.25 \mathrm{~cm}^{2}$, equal to $100 \%$ of the animals' average White Square area). Substrates were computer-generated and laminated to be waterproof. A single 3D object, $6.0 \mathrm{~cm}$ in diameter (approximately equal to $1 \mathrm{ML}$ ) and $6.0 \mathrm{~cm}$ high, was constructed using the same black and white checkerboard squares used for the substrate floor and wall.

\section{Control experiments}

Two control experiments were performed, during which (1) a uniform, $50 \%$ gray substrate floor and 25 -cm-diameter arena wall were used to elicit Uniform patterning; and (2) a checkerboard substrate floor and arena wall were used to elicit Disruptive patterning. For both control experiments, 


\section{A. Experiment 1: Horizontal floor}

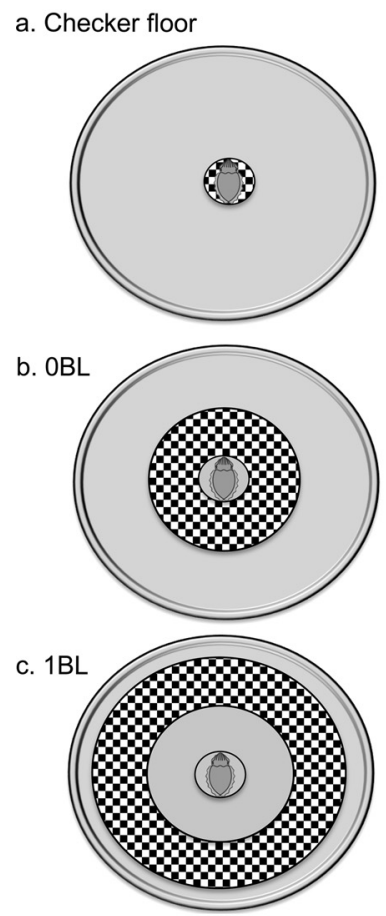

B. Experiment 2: Vertical wall

a. $\mathrm{OBL}$

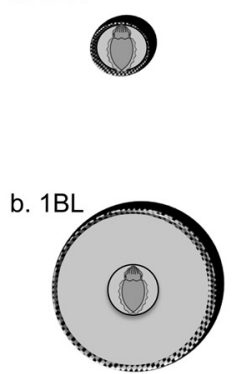

C. Experiment 3: Object

a. $\mathrm{OBL}$

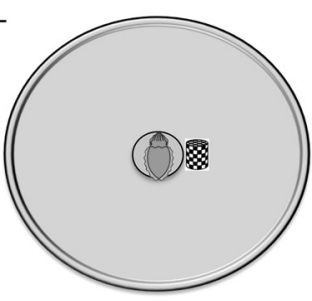

b. $1 \mathrm{BL}$
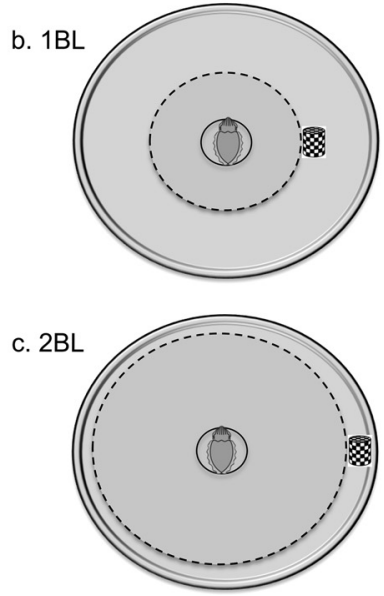

Figure 1. Experimental setup based on the known response of cuttlefish to produce Disruptive body patterns in response to checkerboards of the appropriate size and contrast. Cuttlefish were placed inside a clear plexiglass cylinder and presented with either: (A) a black and white checkerboard floor placed a) directly beneath the cuttlefish, b) at 0 body lengths (BL) away from the cuttlefish, or c) 1 BL away from the cuttlefish; (B) a vertical black and white checkerboard wall placed a) at $0 \mathrm{BL}$ away from the cuttlefish, or b) $1 \mathrm{BL}$ away from the cuttlefish; or (C) a black and white, 3D cylinder placed at a) 0 BL away from the cuttlefish, b) 1 BL away from the cuttlefish, or c) 2 BL away from the cuttlefish.

the walls were presented directly against the exterior of the plexiglass cylinder (at $0 \mathrm{BL}$ ).

\section{Experiment 1: Horizontal floor (background matching)}

(See Fig. 1A for experimental design.) Cuttlefish were presented with high-contrast checkerboard squares located on the 2D floor at three distances: a) directly underneath the cuttlefish in a 1BL-diameter circle; b) directly outside the OBL diameter of the plexiglass cylinder, with a $50 \%$ gray floor located underneath the cuttlefish; and c) a ring around the cuttlefish at $1 \mathrm{BL}(8.0 \mathrm{~cm}$ from plexiglass), with a $50 \%$ gray floor located directly beneath the cuttlefish, extending to the 1BL distance. For Experiment 1, a 50\% gray wall was placed along the edge of the experimental arena.

\section{Experiment 2: Vertical wall (background matching)}

(See Fig. 1B for experimental design.) Cuttlefish were presented with high-contrast checks located on the $2 \mathrm{D}$ vertical wall at two distances: a) at $0 \mathrm{BL}$, right against the clear cylinder; and b) at $1 \mathrm{BL}(8.0 \mathrm{~cm}$ from the plexiglass $)$. For
Experiment 2, a 50\% gray floor covered the entire experimental arena.

\section{Experiment 3: 3D object (masquerade)}

(See Fig. 1C for experimental design.) A high-contrast, 3D object was moved to three distances from the cuttlefish: a) $0 \mathrm{BL}$, i.e., right against the plexiglass cylinder; b) $1 \mathrm{BL}$ ( $8.0 \mathrm{~cm}$ from the plexiglass); and c) $2 \mathrm{BL}$ ( $16.0 \mathrm{~cm}$ from the plexiglass). For Experiment 3, a 50\% gray wall was placed along the edge of the experimental arena, and a 50\% gray floor covered the entire experimental arena.

\section{Image analysis}

We used a MATLAB R2010a-generated image analysis program (The Mathworks, Inc., Natick, MA), developed by C. Chiao, C. Chubb, and L. Siemann, as an automated method for characterizing and discriminating between cuttlefish body patterns (for more detail, see Chiao et al., 2009). This program performs a fast Fourier transform of each image and analyzes the image in different spatial frequency bands, assigning an energy level to each of the six 


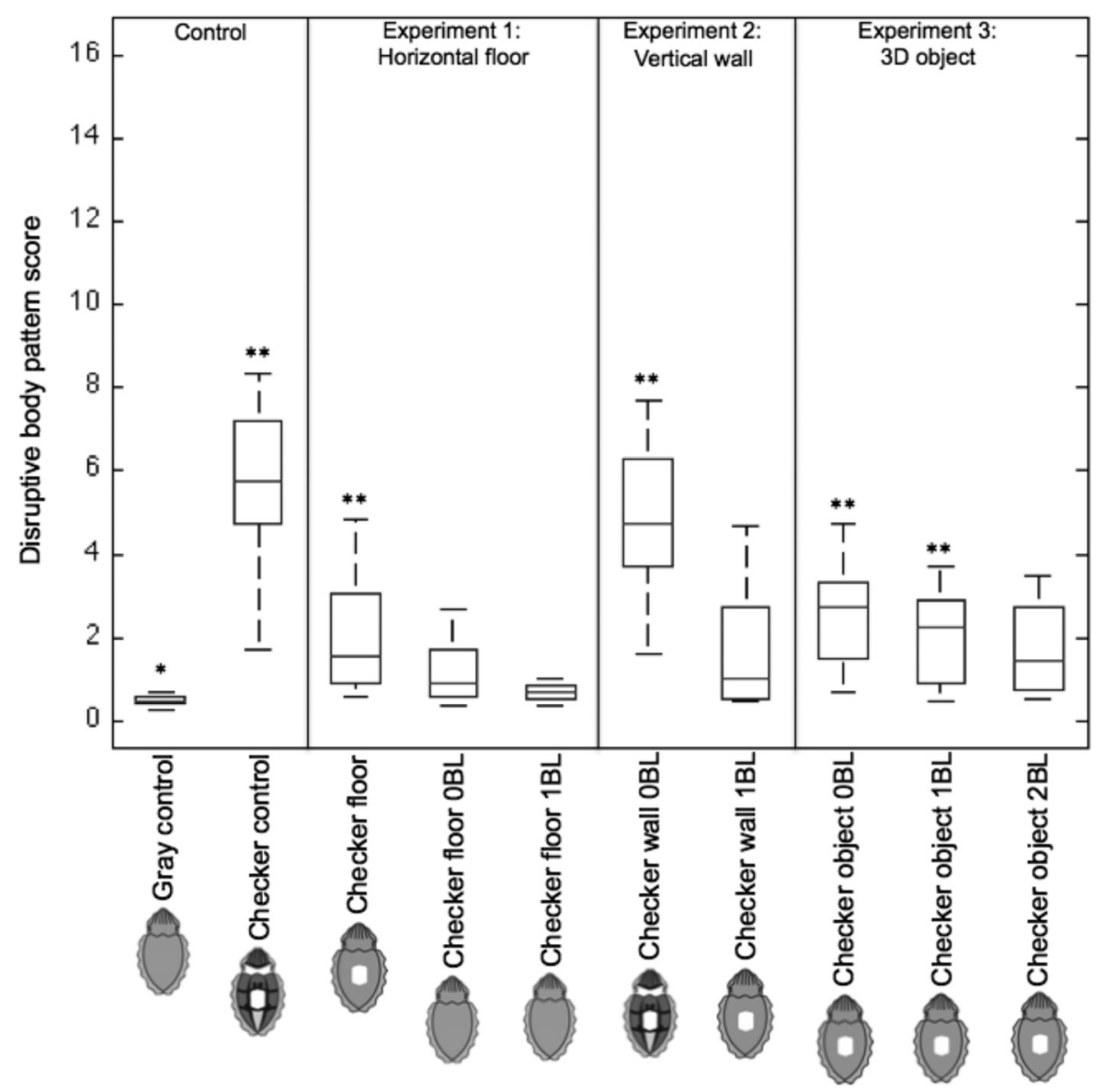

Figure 2. Body patterning responses to each experimental background (see Fig. 1). The Disruptive score is determined by the relative contrast between pixels within each image. Box plots represent the range of Disruptive body pattern scores, the line depicts the median, and error bars are S.D. Two asterisks (**) indicate which substrates differed significantly from the gray control $(*)$. The cuttlefish drawings illustrate the body pattern elicited by each visual background. BL, body length; Checker, checkerboard.

bands. The three cuttlefish body patterns: Uniform, Mottle, and Disruptive, differ in spatial scale (or granularity), and can be distinguished by the distinct shape of their granularity spectra. In addition, this computer program uses landmarks (assigned by the user) on the cuttlefish body to locate 11 Disruptive body pattern components within the image. A "Disruptive pattern score," based on the relative contrast of the pixels in each component, is then generated for each image.

Statistical differences in Disruptive pattern scores by substrate were analyzed using a one-way repeated measures ANOVA in the MATLAB statistics toolbox. Pairwise comparisons between substrates were made using a TukeyKramer test in the multcompare function in MATLAB.

\section{Results}

\section{Control experiments}

A one-way repeated measures ANOVA showed a significant difference in body patterning between substrates $(F=$
22.99; $P=2.76$ e-25). Cuttlefish showed a Uniform body pattern on the $50 \%$ gray control and a Disruptive body pattern on the checkerboard control (Fig. 2). Pairwise comparisons from a Tukey-Kramer test showed a significant difference between the Disruptive body pattern score on the gray control substrate versus the checkerboard control [gray control: $\mathrm{M}$ (mean) $=0.50$, checkerboard control: $\mathrm{M}=6.4$; $P<0.05]$.

\section{Experiment 1: Horizontal floor}

Cuttlefish responded to high-contrast checkerboard squares located on the substrate directly beneath them with weak Disruptive body patterning (i.e., White Square only), but did not respond with Disruptive coloration to checkerboard squares located beyond their immediate $0 \mathrm{BL}$ annulus (Fig. 2). Pairwise comparisons showed a significant difference between the Disruptive body pattern score on the gray control substrate and the checkerboard floor placed directly 
beneath the cuttlefish (gray control: $\mathrm{M}=0.50$, checkerboard floor: $\mathrm{M}=2.43 ; P<0.05$ ), but not between the body pattern response on the gray control and the response to the floor when it was $0 \mathrm{BL}$ and $1 \mathrm{BL}$ away $(0 \mathrm{BL}: \mathrm{M}=1.21$, $1 \mathrm{BL}=0.86 ; P>0.05)$.

\section{Experiment 2: Vertical wall}

Animals responded to high-contrast, vertical checkerboard squares located at 0BL with a Disruptive body pattern. The Disruptive response decreased markedly at $1 \mathrm{BL}$ (Fig. 2); in some cuttlefish, only the White Square was expressed and others were Uniform. Pairwise comparisons showed a significant difference between the Disruptive body pattern score on the gray control and the checkerboard wall located at $0 \mathrm{BL}$ (checkerboard wall $0 \mathrm{BL}: \mathrm{M}=4.71$; $P<0.05$ ), but not between the body pattern responses to the gray control and the checkerboard wall at $1 \mathrm{BL}$ (checkerboard wall 1BL: $\mathrm{M}=1.61 ; P>0.05)$.

\section{Experiment 3: 3D object}

Animals responded with weak Disruptive body patterning (i.e., White Square only) to a high-contrast, 3D object placed at OBL and 1BL (Fig. 2). When the checkerboard object was placed at 2BL, some animals continued to respond with weak Disruptive body patterning, while others became Uniform (Fig. 2). Pairwise comparisons showed a significant difference between the Disruptive body pattern score on the gray control and the checkerboard object located at $0 \mathrm{BL}$ and $1 \mathrm{BL}$ (checkerboard object $\mathrm{OBL}: \mathrm{M}=2.49$; checkerboard object 1BL: $\mathrm{M}=2.21 ; P<0.05$ ), but not between the body pattern response to the gray control and the checkerboard object at 2BL (checkerboard object 2BL: $\mathrm{M}=1.75 ; P>0.05)$.

\section{Discussion}

In these experiments, we set out to address how far away visual stimuli are relevant to cuttlefish camouflage body patterning, and to assess whether there was a difference in their response to 2D horizontal and vertical stimuli. In general, cuttlefish responded with Disruptive body pattern elements only to visual stimuli that were within one body length of distance, whether the stimuli were presented horizontally or vertically. Disruptive body pattern response diminished quickly as stimuli were presented at distances greater than one body length. In addition, the cuttlefish Disruptive body pattern response was strongest in response to both 2D and 3D vertical stimuli than to benthic, horizontal stimuli. This result was not unexpected, since work from our laboratory has shown that vertical stimuli have more influence over cuttlefish body patterning than horizontal stimuli (Barbosa et al., 2008a; Ulmer et al., 2013).
Cuttlefish depend on their unique camouflage abilities for survival. Since their marine environment is often complex and heterogeneous in nature, they must be able rapidly to alter their camouflage body patterning to blend into their surroundings, or to masquerade as nearby objects (e.g., rocks, algae). There are two possible explanations for their use of visual stimuli that are very close by. First, it would seem most effective, in deceiving predator vision, for their camouflage to blend with immediately adjacent backgrounds. Second, as a benthic species, cuttlefish probably experience low-visibility conditions on a regular basis. Since cuttlefish have large and sensitive eyes (Groeger et al., 2005), and can camouflage themselves in extremely low-light conditions (Allen et al., 2010b; Buresch et al., 2015), it is unlikely that their vision is affected much by turbid water. However, the vision of many potential fish predators may be affected by water turbidity (Utne-Palm, 2002). Cuttlefish thus may choose to resemble only nearby objects and substrates, because it is likely they are detected only when a predator is near.

Surprisingly, cuttlefish responded to 3D objects farther away than they did to 2D substrates. This finding suggests that the visual sampling rules for masquerade may differ from those used in background matching. While cuttlefish responded only to $2 \mathrm{D}$ substrates that were very close by (i.e., $0 \mathrm{BL}$ from the animal), they continued to respond to 3D objects when these were placed 2BL from the animal. These results suggest that cuttlefish are more likely to use masquerade than background matching as a camouflage tactic if a high-contrast, 3D object is within a few body lengths away. One reason may be that patterns on the substrate become too distorted for the cuttlefish to recognize when observing them at a near grazing incidence. It is not possible to prove definitively that the camouflage tactic used by the cuttlefish in our experiments was masquerade; such proof would require viewing the cuttlefish from the perspective of a predator (Stevens and Merilaita, 2009; Skelhorn et al., 2010). However, the behavior was similar to that of cuttlefish in the wild - using body posture and texture plus chromatic body patterning to resemble inanimate objects in their surroundings (Hanlon et al., 2009). This finding fits the recent definition of masquerade: "looking like an inedible or inanimate object" (Skelhorn et al., 2010, 2011; Skelhorn and Ruxton, 2011). Other cephalopods use this type of masquerade for camouflage, selectively sampling just a few visual features from their surroundings for body patterning (Hanlon et al., 1999). Two octopus species, Octopus cyanea and Octopus vulgaris, base some of their body patterns on features of nearby objects rather than on their entire field of view (Josef et al., 2012).

Another interesting result of our experiments was that cuttlefish appeared to be able to perceive the distance of the $3 \mathrm{D}$ objects. Camouflage body patterning is generally scaledependent: when the background scale changes, cuttlefish 
deploy an appropriately scaled body pattern. That is, largescale elements elicit Disruptive body patterns; mediumscale elements bring out Mottled body patterns; and small-scale elements, Uniform body patterns (Barbosa et al., 2008b; Chiao et al., 2009). The cuttlefish in this series of experiments appeared to identify the scale of the patterns on the objects presented to them, as noted by their use of a Disruptive body pattern in response to the large-scale checkerboard object. In some cases, they used Disruptive body patterning even when the object was up to $2 \mathrm{BL}$ away. At this distance, the change in perspective would have made the checkerboard squares appear to be $25 \%$ of their original size at OBL. This checkerboard square size $(25 \%$ of the cuttlefish's own White Square area) would normally elicit a Mottle body pattern if presented directly below or beside a cuttlefish (checkerboard squares $12 \%-40 \%$ of an animal's White Square size elicit a Mottle body pattern; Barbosa et al., 2008b). However, cuttlefish responded with a Disruptive body pattern. It is unclear if the apparent ability of cuttlefish to assess the distance of the 3D objects in this experiment-and therefore the actual size of the checkerboard squares-was due to the 3D nature of the object itself or to some other mechanism in the cuttlefish eye.

Recent studies suggest that cuttlefish possess depth perception capability (Josef et al., 2014). Yet since their eyes are laterally placed, they must be able to accommodate in some way the depth necessary to recognize that objects are located at a distance. Two theories have been offered: accommodation is achieved by movement of the lens perpendicular to the axis of the eye (Schaeffel et al., 1999); and, in certain circumstances, the "W" shape of the cuttlefish pupil may aid in depth perception (Mäthger et al., 2013). Regardless, the cuttlefish in our experiments appeared to judge the distance of the $3 \mathrm{D}$ objects that were presented to them. In addition, there is evidence that cuttlefish perceive 3D substrates differently from 2D substrates, and that visual depth may actually increase the strength of some Disruptive components (Kelman et al., 2008), including the White Square- the Disruptive component elicited by the objects in this set of experiments. It is possible that the visual depth of the 3D objects may be the ultimate cause of the difference in cuttlefish response to the $2 \mathrm{D}$ versus $3 \mathrm{D}$ cues that we noted in our experiments.

In the wild, cuttlefish live in a wide variety of natural environments that contain many different objects with visual depth (e.g., rocks, algae, and coral; Hanlon and Messenger, 1988; Hanlon et al., 2011). They have often been observed to camouflage themselves as these objects, even when they are at a distance of five body lengths away (R. Hanlon, pers. obs.). On the other hand, cuttlefish generally only use background matching to camouflage to substrates that are immediately below them or in which they are partially buried (Hanlon and Messenger 1988; Hanlon et al., 1999, 2009; Allen et al., 2010a). These experiments, along with many field observations, highlight the importance of masquerade as a choice of camouflage tactic in this species.

\section{Acknowledgments}

We thank the animal care staff in the Marine Resources Center for help with weekend care of our cuttlefish colony. Liese Siemann and Justine Allen provided valuable insight and discussion for this study. Charlie Chubb provided help with statistics. This work was funded by DARPA/DSO grant no. W15P7T-13-D-CT04.

\section{Literature Cited}

Allen, J. J., L. M. Mäthger, A. Barbosa, and R. T. Hanlon. 2009. Cuttlefish use visual cues to control 3-dimensional skin papillae for camouflage. J. Comp. Physiol. A 195: 547-555.

Allen, J. J., L. M. Mäthger, A. Barbosa, K. C. Buresch, E. Sogin, J. Schwartz, C. Chubb, and R. T. Hanlon. 2010a. Cuttlefish dynamic camouflage: responses to substrate choice and integration of multiple visual cues. Proc. R. Soc. B 277: 1031-1039.

Allen, J. J., L. M. Mäthger, K. C. Buresch, T. Fetchko, M. Gardner, and R. T. Hanlon. 2010b. Night vision by cuttlefish enables changeable camouflage. J. Exp. Biol. 213: 3953-3960.

Barbosa, A., L. M. Mäthger, C. Chubb, C. Florio, C.-C. Chiao, and R. T. Hanlon. 2007. Disruptive coloration in cuttlefish: a visual perception mechanism that regulates ontogenetic adjustment of skin patterning. J. Exp. Biol. 210: 1139-1147

Barbosa, A., L. Litman, and R. T. Hanlon. 2008a. Changeable cuttlefish camouflage is influenced by horizontal and vertical aspects of the visual background. J. Comp. Physiol. A 194: 405-413.

Barbosa, A., L. M. Mäthger, K. C. Buresch, J. Kelly, C. Chubb, C.-C. Chiao, and R. T. Hanlon. 2008b. Cuttlefish camouflage: the effects of substrate contrast and size in evoking uniform, mottle or disruptive body pattern. Vision Res. 48: 1242-1253.

Barbosa, A., J. J. Allen, L. M. Mäthger, and R. T. Hanlon. 2012. Cuttlefish use visual cues to determine arm postures for camouflage. Proc. R. Soc. B 279: 84-90.

Boletzky, S. V. 1983. Sepia officinalis. Pp. 31-52 in Cephalopod Life Cycles, Vol. 1, P. R. Boyle, ed. Academic Press, London.

Buresch, K. C., L. M. Mäthger, J. J. Allen, C. Bennice, N. Smith, J. Schram, C.-C. Chiao, C. Chubb, and R. T. Hanlon. 2011. The use of background matching vs. masquerade for camouflage in cuttlefish Sepia officinalis. Vision Res. 51: 2362-2368.

Buresch, K. C., K. M. Ulmer, D. Akkaynak, D. Allen, J. J. Mäthger, L. M. Nakamura, and R. T. Hanlon. 2015. Cuttlefish adjust body pattern intensity with respect to substrate intensity to aid camouflage, but do not camouflage in extremely low light. J. Exp. Mar. Biol. Ecol. 462: $121-126$.

Chiao, C.-C., and R. T. Hanlon. 2001a. Cuttlefish camouflage: visual perception of size, contrast and number of white squares on artificial checkerboard substrata initiates disruptive coloration. J. Exp. Biol. 204: 2119-2125.

Chiao, C.-C., and R. T. Hanlon. 2001b. Cuttlefish cue visually on area-not shape or aspect ratio- of light objects in the substrate to produce disruptive body patterns for camouflage. Biol. Bull. 201: $269-270$.

Chiao, C.-C., E. J. Kelman, and R. T. Hanlon. 2005. Disruptive body patterning of cuttlefish (Sepia officinalis) requires visual information regarding edges and contrast of objects in natural substrate backgrounds. Biol. Bull. 208: 7-11.

Chiao, C.-C., C. Chubb, and R. T. Hanlon. 2007. Interactive effects of size, contrast, intensity and configuration of background objects in 
evoking disruptive camouflage in cuttlefish. Vision Res. 47: 22232235.

Chiao, C.-C., C. Chubb, K. C. Buresch, and L. Siemann. 2009. The scaling effects of substrate texture on camouflage patterning in cuttlefish. Vision Res. 49: 1647-1656.

Chiao, C.-C., C. Chubb, K. C. Buresch, J. J. Allen, A. Barbosa, L. M. Mäthger, and R. T. Hanlon. 2010. Mottle camouflage patterns in cuttlefish: quantitative characterization and visual stimuli that evoke them. J. Exp. Biol. 213: 187-199.

Chiao, C.-C., K. M. Ulmer, L. A. Siemann, K. C. Buresch, C. Chubb, and R. T. Hanlon. 2013. How visual edge features influence cuttlefish camouflage patterning. Vision Res. 83: 40-47.

Cott, H. B. 1940. Adaptive Coloration in Animals. Methuen, London.

Groeger, G., P. A. Cotton, and R. Williamson. 2005. Ontogenetic changes in the visual acuity of Sepia officinalis measured using the optomotor response. Can. J. Zool. 83: 274-279.

Hanlon, R.T. 2007. Cephalopod dynamic camouflage. Curr. Biol. 17: 400-404.

Hanlon, R. T., and J. B. Messenger. 1988. Adaptive coloration in young cuttlefish (Sepia officinalis L.): the morphology and development of body patterns and their relation to behaviour. Philos. Trans. $R$. Soc. Lond. B 320: 437-487.

Hanlon, R. T., and J. B. Messenger. 1996. Cephalopod Behaviour. Cambridge University Press, Cambridge.

Hanlon, R. T., J. W. Forsythe, and D. E. Joneschild. 1999. Crypsis, conspicuousness, mimicry and polyphenism as antipredator defences of foraging octopuses on Indo-Pacific coral reefs, with a method of quantifying crypsis from video tapes. Biol. J. Linn. Soc. 66: 1-22.

Hanlon, R. T., C.-C. Chiao, L. M. Mäthger, A. Barbosa, K. C. Buresch, and C. Chubb. 2009. Cephalopod dynamic camouflage: bridging the continuum between background matching and disruptive coloration. Philos. Trans. R. Soc. Lond. B 364: 429-437.

Hanlon, R. T., C.-C. Chiao, L. M. Mäthger, K. C. Buresch, A. Barbosa, J. J. Allen, L. Siemann, and C. Chubb. 2011. Rapid adaptive camouflage in cephalopods. Pp. 145-163 in Animal Camouflage: Mechanisms and Functions, M. Stevens and S. Merilaita, eds. Cambridge University Press, Cambridge.

Holmes, W. 1940. The colour changes and colour patterns of Sepia officinalis L. Proc. Zool. Soc. Lond. A 110: 2-35.

Josef, N., P. Amodio, G. Fiorito, and N. Shashar. 2012. Camouflaging in a complex environment-octopuses use specific features of their surroundings for background matching. PLoS One 7: e37579.

Josef, N., O. Mann, A. V. Sykes, G. Fiorito, J. Reis, S. Maccusker, and N. Shashar. 2014. Depth perception: cuttlefish (Sepia officinalis) respond to visual texture density gradients. Anim. Cogn. 14: 13931400.

Kelman, E. J., R. J. Baddeley, A. J. Shohet, and D. Osorio. 2007. Perception of visual texture and the expression of disruptive camouflage by the cuttlefish, Sepia officinalis. Proc. R. Soc. B 274: 13691375 .
Kelman, E. J., D. Osorio, and R. J. Baddeley. 2008. A review of cuttlefish camouflage and object recognition and evidence for depth perception. J. Exp. Biol. 211:1757-1763.

Marshall, N. J., and J. B. Messenger. 1996. Colour-blind camouflage. Nature 382: 408-409.

Mäthger, L. M., A. Barbosa, S. Miner, and R. T. Hanlon. 2006. Color blindness and contrast perception in cuttlefish (Sepia officinalis) determined by a visual sensorimotor assay. Vision Res. 46: 1746-1753.

Mäthger, L. M., C.-C. Chiao, A. Barbosa, K. C. Buresch, S. Kaye, and R. T. Hanlon. 2007. Disruptive coloration elicited on controlled natural substrates in cuttlefish, Sepia officinalis. J. Exp. Biol. 210: 2657-2666.

Mäthger, L. M., R. T. Hanlon, J. Håkansson, and D.-E. Nilsson. 2013. The W-shaped pupil in cuttlefish (Sepia officinalis): functions for improving horizontal vision. Vision Res. 83: 19-24.

Schaeffel, F., C. J. Murphy, and H. C. Howland. 1999. Accommodation in the cuttlefish (Sepia officinalis). J. Exp. Biol. 202: 3127-3134.

Shohet, A. J., R. J. Baddeley, J. C. Anderson, E. J. Kelman, and D. Osorio. 2006. Cuttlefish responses to visual orientation of substrates, water flow and a model of motion camouflage. J. Exp. Biol. 209: 4717-4723.

Shohet, A. J., R. J. Baddeley, J. C. Anderson, and D. Osorio. 2007. Cuttlefish camouflage: a quantitative study of patterning. Biol. J. Linn. Soc. 92: 335-345.

Skelhorn, J., and G. D. Ruxton. 2011. Mimicking multiple models: polyphenetic masqueraders gain additional benefits from crypsis. $B e-$ hav. Ecol. 22: 60-65.

Skelhorn, J., H. M. Rowland, and G. D. Ruxton. 2010. The evolution and ecology of masquerade. Biol. J. Linn. Soc. 99: 1-8.

Skelhorn, J., H. M. Rowland, J. Delf, M. P. Speed, and G. D. Ruxton. 2011. Density-dependent predation influences the evolution and behavior of masquerading prey. Proc. Natl. Acad. Sci. USA 108: 65326536

Stevens, M., and S. Merilaita. 2009. Animal camouflage: current issues and new perspectives. Philos. Trans. R. Soc. Lond. B 364: 423-427.

Ulmer, K. M., K. C. Buresch, M. M. Kossodo, L. M. Mäthger, L. A. Siemann, and R. T. Hanlon. 2013. Vertical visual features have a strong influence on cuttlefish camouflage. Biol. Bull. 224: 110-118.

Utne-Palm, A. C. 2002. Visual feeding of fish in a turbid environment: physical and behavioural aspects. Mar. Freshw. Behav. Physiol. 35: 111-128.

Zylinski, S., D. Osorio, and A. J. Shohet. 2009a. Edge detection and texture classification by cuttlefish. J. Vision 9: 1-10.

Zylinski, S., D. Osorio, and A. J. Shohet. 2009b. Perception of edges and visual texture in the camouflage of the common cuttlefish, Sepia officinalis. Philos. Trans. R. Soc. Lond. B 364: 439-448.

Zylinski, S., D. Osorio, and A. J. Shohet. 2009c. Cuttlefish camouflage: context-dependent body pattern use during motion. Proc. R. Soc. B 276: $3963-3969$. 